\title{
Minimum Cost Input/Output Design for Large-Scale Linear Structural Systems
}

\author{
Sérgio Pequito ${ }^{\mathrm{a}, \mathrm{b}}$, Soummya Kar ${ }^{\mathrm{a}}$, A. Pedro Aguiar ${ }^{\mathrm{c}}$ \\ ${ }^{a}$ Department of Electrical and Computer Engineering, Carnegie Mellon University, Pittsburgh, PA 15213 \\ ${ }^{\mathrm{b}}$ Institute for Systems and Robotics, Instituto Superior Técnico, Technical University of Lisbon, Lisbon, Portugal \\ ${ }^{\mathrm{c}}$ Department of Electrical and Computer Engineering, Faculty of Engineering, University of Porto, Porto, Portugal
}

\begin{abstract}
In this paper, we provide optimal solutions to two different (but related) input/output design problems involving large-scale linear dynamical systems, where the cost associated to each directly actuated/measured state variable can take different values, but is independent of the input/output performing the task. Under these conditions, we first aim to determine and characterize the input/output placement that incurs in the minimum cost while ensuring that the resulting placement achieves structural controllability/observability. Further, we address a constrained variant of the above problem, in which we seek to determine the minimum cost placement configuration, among all possible input/output placement configurations that ensures structural controllability/observability, with the lowest number of directly actuated/measured state variables. We develop new graphtheoretical characterizations of cost-constrained input selections for structural controllability and properties that enable us to address both problems by reduction to a weighted maximum matching problem - efficiently addressed by algorithms with polynomial time complexity (in the number of state variables). Finally, we illustrate the obtained results with an example.
\end{abstract}

Key words: Linear Structural Systems, Input/Output Selection, Graph Theory, Computational Complexity

\section{Introduction}

The problem of control systems design, meeting certain desired specifications, is of fundamental importance. Possible specifications include (but are not restricted to) controllability and observability. These specifications ensure the capability of a dynamical system (such as chemical process plants, refineries, power plants, and airplanes, to name a few) to drive its state toward a specified goal or infer its present state. To achieve these specifications, the selection of where to place the actuators and sensors assumes a critical importance. More often than not, we need to consider the cost per actuator/sensor, that depends on its specific functionality and/or its installation and maintenance cost. The resulting placement cost optimization problem (apparently combinatorial) can be quite non-trivial, and currently applied state-of-the-art methods typically consider relaxations of the optimization problem, brute force approaches or heuristics, see for instance Padula and Kincaid (1999); Frecker (2003); Begg and Liu (2000);

^ This work was partially supported by grant SFRH/BD/33779/2009, from Fundação para a Ciência e a Tecnologia (FCT), the CMU-Portugal (ICTI) program, and NSF grant 1306128.

Email addresses: sergio.pequito@gmail.com (Sérgio Pequito), soummyak@andrew.cmu.edu (Soummya Kar), pedro.aguiar@fe.up.pt (A. Pedro Aguiar).
Chmielewski et al. (2002); Fahroo and Demetriou (2000).

An additional problem is the fact that the precise numerical values of the system model parameters are generally not available for many large-scale systems of interest. A natural direction is to consider structural systems (Dion et al., 2003) based reformulations, which we pursue in this work. Representative work in structural systems theory may be found in Lin (1974); Siljak (2007); Reinschke (1988); Murota (2009), and Liu et al. (2011); Ruths and Ruths (2014) in the context of (structural) controllability and observability studies in complex networks. The main idea is to reformulate and study an equivalence class of systems for which system-theoretic properties are investigated on the basis of the location of zeros and (possibly) nonzeros of the state space representation matrices. Properties such as controllability and observability are, in this framework, referred to as structural controllability and structural observability, respectively. In addition, controllability and observability properties hold for almost all possible of real matrices satisfying the mentioned pattern (Dion et al., 2003).

In this context, consider a given (possibly large-scale) system with autonomous dynamics

$$
\dot{x}=A x,
$$


where $x \in \mathbb{R}^{n}$ denotes the state and $A$ is the $n \times n$ dynamics matrix. Suppose that the sparsity pattern, i.e., location of zeros and (possibly) nonzeros, of $A$ is available, but the specific numerical values of the remaining elements ist not known. Subsequently, let $\bar{A} \in\{0,1\}^{n \times n}$ be the binary matrix that represents the structural pattern of $A$, i.e., it encodes the sparsity pattern of $A$ by assigning 0 to each zero entry of $A$ and 1 otherwise.

Hereafter, we introduce two different (but related) input/output design problems involving large-scale linear dynamical systems, where the cost associated to each directly actuated/measured state variable can take different values, but is independent of the input/output performing the task. These costs can capture the specific functionality required from an actuator and/or its installation and maintenance cost, regarding the actuation of a specific state variables. Under these conditions, we first aim to determine and characterize the input/output placement that incurs in the minimum cost while ensuring that the resulting placement achieves structural controllability/observability as presented in $\mathcal{P}_{2}$. Further, we address a constrained variant of the above problem, in which we seek to determine the minimum cost placement configuration, among all possible input/output placement configurations that ensures structural controllability/observability, with the lowest number of directly actuated/measured state variables (Pequito et al., 2013a) as stated in $\mathcal{P}_{1}$.

\section{Problems Statement}

Given the structure of the dynamics matrix $\bar{A} \in$ $\{0,1\}^{n \times n}$ and a vector $c$ of size $n$, where the entry $c_{i} \geq 0$ denotes the cost of directly actuating the state variable $i$, determine the sparsity of the input matrix $\bar{B}$ that solves the following optimization problems

$$
\begin{array}{cc}
\mathcal{P}_{1}: \quad \min _{\bar{B} \in\{0,1\}^{n \times n}} & \|\bar{B}\|_{c} \\
\text { s.t. } & (\bar{A}, \bar{B}) \text { structurally controllable } \\
& \|\bar{B}\|_{0} \leq\left\|\bar{B}^{\prime}\right\|_{0} \text {, for all } \\
& \left(\bar{A}, \bar{B}^{\prime}\right) \text { structurally controllable, }
\end{array}
$$

and

$$
\begin{array}{cc}
\mathcal{P}_{2}: & \|\bar{B}\|_{c} \\
& \min _{\bar{B} \in\{0,1\}^{n \times n}} \\
\text { s.t. } & (\bar{A}, \bar{B}) \text { structurally controllable. }
\end{array}
$$

where $\|\bar{B}\|_{c}=c^{T} \bar{B} \mathbf{1},\|\bar{B}\|_{0}$ denotes the zero (quasi) norm corresponding to the number of nonzero entries in $\bar{B}$, and 1 the vector of ones with size $n$. Notice that a solution to $\mathcal{P}_{1}$ or $\mathcal{P}_{2}$ may consist of columns with all zero entries, that can be disregarded when considering the deployment of the inputs required to actuate the system. Notice that in the worst case scenario, taking the identity matrix as the input matrix we obtain structural controllability, which justifies the dimensions chosen for the solution search space.
Notice that in problems $\mathcal{P}_{1}$ and $\mathcal{P}_{2}$, some solutions may comprise one nonzero entry in a column; in other words, solutions in which an input actuates one state variable, which we refer to as dedicated inputs. Additionally, if a solution $\bar{B}^{*}$ is such that all its nonzero columns consist of exactly one nonzero entry, then it is referred to as a dedicated solution, otherwise it is referred to as a non-dedicated solution. For instance, in the context of leader-selection problems, it corresponds to determining which agents should receive input signals from an external source. If the signals are crafted for a specific agent, then the input is dedicated, as it is common in peer-to-peer communication schemes. Alternatively, if the signal is broadcasted to a collection of (at least two) agents, the input is not dedicated, since a collection of individuals receive the same signal. In addition, observe that in $\mathcal{P}_{1}$ there is a restriction of obtaining a solution with the minimum number of state variables that need to be directly actuated in order to achieve structural controllability. Without such restriction, i.e., by possibly actuating more state variables, we may obtain a lower cost placement achieving structural controllability, hence, the interest in studying $\mathcal{P}_{2}$. Nonetheless, the constrained scenario in $\mathcal{P}_{1}$ may be desirable, for instance, in multi-agent networks in an environment where communication (of the input signal) is very expensive in comparison with actuation cost of a specific agent, or a collection of state variables for dynamical systems at large.

Finally, note that the solution procedures for $\mathcal{P}_{1}$ and $\mathcal{P}_{2}$ also address the corresponding structural observability output matrix design problem by invoking the duality between observability and controllability in linear timeinvariant (LTI) systems (Hespanha, 2009).

Recently, the I/O selection problem have received increasing attention in the literature: the minimal controllability problem, i.e., the problem of determining the sparsest input matrix that ensures controllability of a given LTI system (Olshevsky, 2014; Ramos et al., 2014), and in Summers et al. (2015); Tzoumas et al. (2015); Clark et al. (2014); Clark and Poovendran (2011); Pasqualetti et al. (2014); Lin et al. (2014) the configuration of actuators is sought to ensure certain performance criteria, for instance, by optimizing properties of the controllability Grammian.

Alternatively, I/O selection problem for structural linear systems has also been addressed in Commault and Dion (2013); Dion et al. (2003); Pequito et al. (2015a, 2013b,c,a, 2015b); Liu et al. (2011); Ruths and Ruths (2014) and references therein, just to name a few. In particular, in Pequito et al. (2015a), the structural version of the minimal controllability problem, or the minimal structural controllability problem, was shown to be polynomially solvable; an improvement on the computational complexity was analyzed in detail for several subsystems in Assadi et al. (2015). Notice that this is a particular instance of $\mathcal{P}_{1}$ and $\mathcal{P}_{2}$ when the costs are uniform, i.e., 
each variable incurs in the same (non-zero) cost.

The solution proposed in Pequito et al. (2015a) provides useful insights, but is not sufficient to address the problems $\mathcal{P}_{1}$ and $\mathcal{P}_{2}$ with non-uniform cost. Nonetheless, the characterizations obtained in Pequito et al. (2015a) were used to obtain some preliminary results on problems $\mathcal{P}_{1}$ and $\mathcal{P}_{2}$ in Pequito et al. (2013a) and Pequito et al. (2013c), respectively. These preliminary results are based on analyzing the intrinsic properties of the class of all minimal subsets of state variables that need to be actuated by dedicated inputs to ensure structural controllability; in particular, the proposed solution provided algorithmic solutions with computational time complexity $\mathcal{O}\left(n^{3.5}\right)$, as a result of evaluating $n$ maximum matchings using the Hungarian algorithm Cormen et al. (2001). In addition, in Olshevsky (2015) the problem $\mathcal{P}_{1}$ is addressed for a specific binary actuation cost structure $c \in\{0, \infty\}^{n}$, and a solution with computational time complexity $\mathcal{O}(n+m \sqrt{n})$ is proposed, where $m$ denotes the total number of non-zero entries, and $\mathcal{O}\left(n^{2.5}\right)$ in general. Similarly, although (Olshevsky, 2015) provides useful insights to address $\mathcal{P}_{1}$, it is not sufficient to address the problems $\mathcal{P}_{1}$ with non-uniform cost, as well as $\mathcal{P}_{2}$.

The main contributions of this paper are as follows: by presenting new graph-theoretical characterizations of cost-constrained input selections for structural controllability and results on the properties of weighted maximum matchings, we can cast both $\mathcal{P}_{1}$ and $\mathcal{P}_{2}$ as a weighted maximum matching problem - a well known graph-theoretic algorithm that can be efficiently addressed by algorithms with polynomial time complexity (in the number of state variables); hence, leading to algorithms with computational time complexity $\mathcal{O}\left(n^{\omega}\right)$ (Mucha and Sankowski, 2004), where $\omega<2.373$ is the lowest exponent known associated with the complexity of multiplying two $n \times n$ matrices. Notwithstanding, the minimum weighted maximum matching can be arbitrarily approximated by executing linear computational complexity algorithms (Duan and Pettie, 2014), thus linear time algorithms for obtaining approximate solutions of $\mathcal{P}_{1}$ and $\mathcal{P}_{2}$ arbitrarily close to the optimal are readily available. In addition, the proposed solutions are obtained while exploring the relation between these two problems; more specifically, we use insights from $\mathcal{P}_{1}$ to solve $\mathcal{P}_{2}$, which sheds light on possible extensions towards less restrictive cost assumptions. More recently, in ? the problem was extended to the case where a state variable has a cost that depends on the input that actuates it, hence, leading to a multiple heterogenous cost scenario. Alternatively, the problem of determining the minimum number of actuators from a given collection of possible actuator-state configurations was shown to be (in general) NP-hard Pequito et al. (2015b). Notwithstanding, in ? it was shown that the same problem can be polynomially solvable when the dynamic matrix is irreducible.

The rest of the paper is organized as follows: Section 2 reviews results from structural systems and some graph theorectical concepts required to obtain the main results of this paper. Section 3 presents the main results of the paper, in particular, a procedure to determine the minimal cost placement of inputs in LTI systems, as formulated in $\mathcal{P}_{1}$ and $\mathcal{P}_{2}$. Section 4 illustrates the procedures through an example. Finally, Section 5 concludes the paper, and presents avenues for future research.

\section{Preliminaries and Terminology}

The following standard terminology and notions from graph theory can be found, for instance in Pequito et al. $(2015 a)$. Let $\mathcal{D}(\bar{A})=\left(\mathcal{X}, \mathcal{E}_{\mathcal{X}, \mathcal{X}}\right)$ be the digraph representation of $\bar{A} \in\{0,1\}^{n \times n}$, to be referred to as the state digraph, where the vertex set $\mathcal{X}$ represents the set of state variables (also referred to as state vertices) and $\mathcal{E}_{\mathcal{X}, \mathcal{X}}=\left\{\left(x_{i}, x_{j}\right): \bar{A}_{j i} \neq 0\right\}$ denotes the set of edges. Similarly, given $\bar{B} \in\{0,1\}^{n \times p}$, we define the digraph $\mathcal{D}(\bar{A}, \bar{B})=\left(\mathcal{X} \cup \mathcal{U}, \mathcal{E}_{\mathcal{X}, \mathcal{X}} \cup \mathcal{E}_{\mathcal{U}, \mathcal{X}}\right)$, to be referred to as the system digraph, where $\mathcal{U}$ represents the set of input vertices and $\mathcal{E}_{\mathcal{U}, \mathcal{X}}=\left\{\left(u_{i}, x_{j}\right): \bar{B}_{j i} \neq 0\right\}$. Further, by similarity, we have the state-slack digraph given by $\mathcal{D}(\bar{A}, \bar{S})=\left(\mathcal{X} \cup \mathcal{S}, \mathcal{E}_{\mathcal{X}, \mathcal{X}} \cup \mathcal{E}_{\mathcal{S}, \mathcal{X}}\right)$, where $\mathcal{S}$ represents the set of (auxiliary) slack variables (or vertices) that take the role of potential inputs in the solutions proposed to our problems. In addition, given digraphs $\mathcal{D}(\bar{A}, \bar{B})$ and $\mathcal{D}(\bar{A}, \bar{S})$, we say that they are isomorphic to each other, if there exists a bijective relationship between the vertices and edges of the digraphs that preserves the incidence relation. Finally, since the edges are directed, an edge is said to be an outgoing edge from a vertex $v$ if it starts in $v$, and, similarly, is said to be an incoming edge to $w$ if it ends on $w$.

In addition, we will use the following graph theoretic notions Cormen et al. (2001): A digraph $\mathcal{D}_{s}=\left(\mathcal{V}_{s}, \mathcal{E}_{s}\right)$ with $\mathcal{V}_{s} \subset \mathcal{V}$ and $\mathcal{E}_{s} \subset \mathcal{E}$ is called a subgraph of $\mathcal{D}=(\mathcal{V}, \mathcal{E})$. A sequence of edges $\left\{\left(v_{1}, v_{2}\right),\left(v_{2}, v_{3}\right), \cdots,\left(v_{k-1}, v_{k}\right)\right\}$, in which all the vertices are distinct, is called an elementary path from $v_{1}$ to $v_{k}$. A vertex with an edge to itself (i.e., a self-loop), or an elementary path from $v_{1}$ to $v_{k}$ together with an additional edge $\left(v_{k}, v_{1}\right)$, is called a cycle. A digraph $\mathcal{D}$ is said to be strongly connected if there exists a directed path between any two pairs of vertices. A strongly connected component (SCC) is a maximal subgraph $\mathcal{D}_{s}=\left(\mathcal{V}_{s}, \mathcal{E}_{s}\right)$ of $\mathcal{D}$ such that for every $v, w \in \mathcal{V}_{s}$ there exists a path from $v$ to $w$. Notice that the SCCs are uniquely defined for a given digraph; consequently, visualizing each SCC as a virtual node (or supernode), we can generate a directed acyclic graph (DAG), in which each node corresponds to a single SCC and there exists a directed edge between two virtual nodes if and only if there exists a directed edge connecting vertices within the corresponding SCCs in the original digraph. The DAG associated with $\mathcal{D}=(\mathcal{V}, \mathcal{E})$ can be efficiently generated in $\mathcal{O}(|\mathcal{V}|+|\mathcal{E}|)$ (Cormen et al., 2001), where $|\mathcal{V}|$ and $|\mathcal{E}|$ denote the number of vertices in $\mathcal{V}$ and the number of edges in $\mathcal{E}$, respectively. In the DAG representation, an SCC (a supernode) that has no incoming 
edge from any state in a different SCC (supernode) is referred to as a non-top linked $S C C$, since, by convention, the DAG is graphically represented with edges between the virtual nodes drawn downwards.

For any two vertex sets $\mathcal{S}_{1}, \mathcal{S}_{2} \subset \mathcal{V}$, we define the $b i$ partite graph $\mathcal{B}\left(\mathcal{S}_{1}, \mathcal{S}_{2}, \mathcal{E}_{\mathcal{S}_{1}, \mathcal{S}_{2}}\right)$, as a graph (bipartite), whose vertex set is given by $\mathcal{S}_{1} \cup \mathcal{S}_{2}$ and the edge set $\mathcal{E}_{S_{1}, S_{2}} \subseteq\left\{\left(s_{1}, s_{2}\right) \in \mathcal{E}: s_{1} \in \mathcal{S}_{1}, s_{2} \in \mathcal{S}_{2}\right\}$. Given $\mathcal{B}\left(\mathcal{S}_{1}, \mathcal{S}_{2}, \mathcal{E}_{\mathcal{S}_{1}, \mathcal{S}_{2}}\right)$, a matching $M$ corresponds to a subset of edges in $\mathcal{E}_{\mathcal{S}_{1}, \mathcal{S}_{2}}$ that do not share vertices, i.e., given edges $e=\left(s_{1}, s_{2}\right)$ and $e^{\prime}=\left(s_{1}^{\prime}, s_{2}^{\prime}\right)$ with $s_{1}, s_{1}^{\prime} \in \mathcal{S}_{1}$ and $s_{2}, s_{2}^{\prime} \in \mathcal{S}_{2}, e, e^{\prime} \in M$ only if $s_{1} \neq s_{1}^{\prime}$ and $s_{2} \neq s_{2}^{\prime}$. A bipartite graph is, by convention, depicted by a set of vertices $\mathcal{S}_{1}$ in the left and another set of vertices $\mathcal{S}_{2}$ in the right to clearly emphasize the bipartition. The vertices in $\mathcal{S}_{1}$ and $\mathcal{S}_{2}$ are matched vertices if they belong to an edge in the matching $M$, otherwise, we designate the vertices as unmatched vertices. A maximum matching $M^{*}$ is a matching $M$ that has the largest number of edges among all possible matchings. It is to be noted that a maximum matching $M^{*}$ may not be unique. For ease of referencing, keeping in mind the bipartite graphical representation, the term right-unmatched vertices, with respect to (w.r.t.) $\mathcal{B}\left(\mathcal{S}_{1}, \mathcal{S}_{2}, \mathcal{E}_{\mathcal{S}_{1}, \mathcal{S}_{2}}\right)$ and a matching $M$ (not necessarily maximum), will refer to those vertices in $\mathcal{S}_{2}$ that do not belong to a matching edge in $M$, and are denoted by $\mathcal{U}_{R}(M)$. In addition, we introduce the following notation: given a set of edges $\mathcal{E}_{\mathcal{S}_{1}, \mathcal{S}_{2}}$, we denote by $\mathcal{L}\left(\mathcal{E}_{\mathcal{S}_{1}, \mathcal{S}_{2}}\right)$ and $\mathcal{R}\left(\mathcal{E}_{\mathcal{S}_{1}, \mathcal{S}_{2}}\right)$ the collection of vertices corresponding to the set of left and right endpoints of $\mathcal{E}_{\mathcal{S}_{1}, \mathcal{S}_{2}}$, i.e., in $\mathcal{S}_{1}$ and $\mathcal{S}_{2}$, respectively.

Now, we present some specific bipartite graphs that are closely related with the digraphs previously introduced. More precisely, we have: (i) the state bipartite graph $\mathcal{B}(\bar{A})=\mathcal{B}\left(\mathcal{X}, \mathcal{X}, \mathcal{E}_{\mathcal{X}, \mathcal{X}}\right)$ that we often refer to as the bipartite representation of (or associated with, or induced by) the state digraph $\mathcal{D}(\bar{A})$; (ii) the system bipartite graph $\mathcal{B}(\bar{A}, \bar{B})=\mathcal{B}\left(\mathcal{X} \cup \mathcal{U}, \mathcal{X}, \mathcal{E}_{\mathcal{X}, \mathcal{X}} \cup \mathcal{E}_{\mathcal{U}, \mathcal{X}}\right)$ that we often refer to as the bipartite representation of $\mathcal{D}(\bar{A}, \bar{B})$; and, similarly to the latter, we have (iii) the state-slack bipartite graph $\mathcal{B}(\bar{A}, \bar{S})=\mathcal{B}\left(\mathcal{X} \cup \mathcal{S}, \mathcal{X}, \mathcal{E}_{\mathcal{X}, \mathcal{X}} \cup \mathcal{E}_{\mathcal{S}, \mathcal{X}}\right)$ that we often refer to as the bipartite representation of the state-slack digraph $\mathcal{D}(\bar{A}, \bar{S})$.

If we associate weights (or costs) with the edges in a digraph and bipartite graph, we obtain a weighted digraph and weighted bipartite graph, respectively. A weighted digraph is represented by the pair digraph-weight given by $(\mathcal{D}=(\mathcal{V}, \mathcal{E}) ; w)$, where $w: \mathcal{E} \rightarrow \mathbb{R}_{0}^{+} \cup\{\infty\}$ is the weight function. Similarly, a weighted bipartite graph is represented by the pair bipartite-weight $\left(\mathcal{B}\left(\mathcal{S}_{1}, \mathcal{S}_{2}, \mathcal{E}_{\mathcal{S}_{1}, \mathcal{S}_{2}}\right) ; w\right)$. Next, we revisit the minimum weight maximum matching (MWMM) problem (Kuhn, 1955; Munkres, 1957). This problem consists in determining the maximum matching of a weighted bipartite graph $\left(\mathcal{B}\left(\mathcal{S}_{1}, \mathcal{S}_{2}, \mathcal{E}_{\mathcal{S}_{1}, \mathcal{S}_{2}}\right) ; w\right)$ that incurs the minimum weight-sum of its edges; in other words, determining the maximum matching $M^{c}$ such that

$$
M^{c}=\arg \min _{M \in \mathcal{M}} \sum_{e \in M} w(e),
$$

where $\mathcal{M}$ is the set of all maximum matchings of $\mathcal{B}\left(\mathcal{S}_{1}, \mathcal{S}_{2}, \mathcal{E}_{\mathcal{S}_{1}, \mathcal{S}_{2}}\right)$.

We will also require the following general results on structural control design from (Pequito et al., 2015a)). We define a feasible dedicated input configuration to be a collection of state variables to which by assigning dedicated inputs we can ensure structural controllability of the system. Consequently, a minimal feasible dedicated input configuration is the minimal subset of state variables to which we need to assign dedicated inputs to ensure structural controllability. Further, the feasible dedicated input configurations can be characterized as follows.

Theorem 1 (Pequito et al. $(2015 a)$ ). Let $\mathcal{D}(\bar{A})=$ $\left(\mathcal{X}, \mathcal{E}_{\mathcal{X}, \mathcal{X}}\right)$ denote the system digraph and $\mathcal{B}(\bar{A}) \equiv$ $\mathcal{B}\left(\mathcal{X}, \mathcal{X}, \mathcal{E}_{\mathcal{X}, \mathcal{X}}\right)$ the associated state bipartite graph. Let $\mathcal{S}_{u} \subset \mathcal{X}$, then the following statements are equivalent:

(1) The set $\mathcal{S}_{u}$ is a feasible dedicated input configuration;

(2) There exists a subset $\mathcal{U}_{R}\left(M^{*}\right) \subset \mathcal{S}_{u}$ corresponding to the set of right-unmatched vertices of some maximum matching $M^{*}$ of $\mathcal{B}(\bar{A})$, and a subset $\mathcal{A}_{u} \subset \mathcal{S}_{u}$ comprising one state variable from each non-top linked $S C C$ of $\mathcal{D}(\bar{A})$.

Observe that a state variable can be simultaneously in $\mathcal{U}_{R}\left(M^{*}\right)$ and $\mathcal{A}_{u}$, even if these sets correspond to those of a minimal feasible dedicated input configuration; thus, motivating us to refer to those variables as playing a double role, since they contribute to both the conditions in Theorem 1.

Remark 1. In Pequito et al. (2015a) general results were given on structural input selection, in particular on non-dedicated structural input design, i.e., in which the structural input matrix $\bar{B}$ may possess multiple nonzero entries in each column. To ease the presentation, we denote by $m$ the number of right-unmatched vertices in any maximum matching of $\mathcal{B}(\bar{A})$ and by $\beta$ the number of nontop linked $S C C$ s in $\mathcal{D}(\bar{A})$. The following characterization of structural controllability was obtained in Theorem 8 in Pequito et al. (2015a): a pair $(\bar{A}, \bar{B})$ is structurally controllable if and only if there exists a maximum matching of $\mathcal{B}(\bar{A})$ with a set of right-unmatched vertices $\mathcal{U}_{R}$, such that, $\bar{B}$ has (at least) $m$ nonzero entries, one in each of the rows corresponding to the different state variables in $\mathcal{U}_{R}$ and located at different columns, and (at least) $\beta$ nonzero entries, each of which belongs to a row (state variable) corresponding to a distinct non-top linked SCC and located in arbitrary columns.

\section{Main Results}

Despite the fact that problems $\mathcal{P}_{1}$ and $\mathcal{P}_{2}$ seem to be combinatorial, hereafter we show that they can be solved 
using polynomial complexity (in the dimension of the state space) algorithms. To obtain these results, we first present some intermediate results where we characterize the matchings that the bipartite graphs used in the sequel can have (Lemma 1 and Lemma 2). Then, these lemmas are used to characterize the MWMMs that a weighted bipartite graph can have, upon a specific cost structure to be used to solve and characterize the solutions to $\mathcal{P}_{1}$ and $\mathcal{P}_{2}$, see Lemma 3 and Lemma 4 . Lastly, we present the reduction of $\mathcal{P}_{1}$ and $\mathcal{P}_{2}$ to a weighted maximum matching, as provided in Algorithm 1, constrained to the conditions presented in Theorem 2 and Theorem 3, respectively.

Let $\bar{S}$ be a $n \times q$ structural (binary) matrix, and denote by $\mathcal{B}(\bar{A}, \bar{S})$ the state-slack bipartite graph associated with the digraph $\mathcal{D}(\bar{A}, \bar{S})$. Note, by construction, the stateslack digraph $\mathcal{D}(\bar{A}, \bar{S})$ consists of $n+q$ vertices, where the $q$ additional vertices (in comparison with the state digraph $\mathcal{D}(\bar{A}))$ correspond to the slack variables, introduced by $\bar{S}$. Further, by construction, the slack variables only have outgoing edges (associated with the nonzero entries of $\bar{S})$ to the state variables in $\mathcal{D}(\bar{A}, \bar{S})$; in other words, there are no incoming edges into the slack variables. We start by relating maximum matchings of the two bipartite graphs $\mathcal{B}(\bar{A})$ and $\mathcal{B}(\bar{A}, \bar{S})$ that will also help in obtaining better insight and better understanding of the properties of the maximum matchings of the different bipartite graphs.

Lemma 1. Let $\mathcal{B}(\bar{A}, \bar{S})=\mathcal{B}\left(\mathcal{X} \cup \mathcal{S}, \mathcal{X}, \mathcal{E}_{\mathcal{X}, \mathcal{X}} \cup \mathcal{E}_{\mathcal{S}, \mathcal{X}}\right)$ be the state-slack bipartite graph, $\mathcal{B}(\bar{A})=\mathcal{B}\left(\mathcal{X}, \mathcal{X}, \mathcal{E}_{\mathcal{X}, \mathcal{X}}\right)$ and $\mathcal{B}(\bar{S})=\mathcal{B}\left(\mathcal{S}, \mathcal{X}, \mathcal{E}_{\mathcal{S}, \mathcal{X}}\right)$. The following statements hold:

(1) If $M_{\bar{A}}$ and $M_{\bar{S}}$ are matchings of $\mathcal{B}(\bar{A})$ and $\mathcal{B}(\bar{S})$ respectively, and $\mathcal{R}\left(M_{\bar{A}}\right) \cap \mathcal{R}\left(M_{\bar{S}}\right)=\emptyset$, then $M_{\bar{A}, \bar{S}}=$ $M_{\bar{S}} \cup M_{\bar{A}}$ is a matching of $\mathcal{B}(\bar{A}, \bar{S})$; and

(2) If $M_{\bar{A}, \bar{S}}$ is a matching of $\mathcal{B}(\bar{A}, \bar{S})$, then $M_{\bar{A}, \bar{S}}=$ $M_{\bar{S}} \cup M_{\bar{A}}$, where $M_{\bar{A}}=M_{\bar{A}, \bar{S}} \cap \mathcal{E}_{\mathcal{X}, \mathcal{X}}$ and $M_{\bar{S}}=$ $M_{\bar{A}, \bar{S}} \cap \mathcal{E}_{\mathcal{S}, \mathcal{X}}$ are (disjoint) matchings of $\mathcal{B}(\bar{A})$ and $\mathcal{B}(\bar{S})$, respectively.

In particular, $\mathcal{R}\left(M_{\bar{S}}\right) \subset \mathcal{U}_{R}\left(M_{\bar{A}}\right)$, where $\mathcal{U}_{R}\left(M_{\bar{A}}\right)$ is the set of right-unmatched vertices associated with the matching $M_{\bar{A}}$.

Proof. The proof of (1) follows by noticing that, by construction of $\mathcal{B}(\bar{A}, \bar{S})$, we have $\mathcal{L}\left(M_{\bar{A}}\right) \cap \mathcal{L}\left(M_{\bar{S}}\right)=\emptyset$, and by assumption $\mathcal{R}\left(M_{\bar{A}}\right) \cap \mathcal{R}\left(M_{\bar{S}}\right)=\emptyset$, which implies that $M_{\bar{A}, \bar{S}}=M_{\bar{S}} \cup M_{\bar{A}}$ has no edge with common endpoints; in other words, it is a matching of $\mathcal{B}(\bar{A}, \bar{S})=$ $\left(\mathcal{X} \cup \mathcal{S}, \mathcal{X}, \mathcal{E}_{\mathcal{X}, \mathcal{X}} \cup \mathcal{E}_{\mathcal{S}, \mathcal{X}}\right)$, by definition of matching.

On the other hand, the proof of (2) follows by noticing that the edges in $M_{\bar{A}, \bar{S}}$ belong to either $\mathcal{E}_{\mathcal{X}, \mathcal{X}}$ or $\mathcal{E}_{\mathcal{S}, \mathcal{X}}$ and noticing that $M_{\bar{A}}$ and $M_{\bar{S}}$ have no common endpoints since $M_{\bar{A}, \bar{S}}$ is a matching. Subsequently, it is easy to see that $M_{\bar{A}}$ and $M_{\bar{S}}$ are matchings of $\mathcal{B}(\bar{A})$ and $\mathcal{B}(\bar{S})$, respectively.

Subsequently, from Lemma 1, we can obtain the following result characterizing the maximum matchings of $\mathcal{B}(\bar{A}, \bar{S})$.

Lemma 2. Let $\mathcal{B}(\bar{A}, \bar{S})=\mathcal{B}\left(\mathcal{X} \cup \mathcal{S}, \mathcal{X}, \mathcal{E}_{\mathcal{X}, \mathcal{X}} \cup \mathcal{E}_{\mathcal{S}, \mathcal{X}}\right)$ be the state-slack bipartite graph. If $M_{\bar{A}, \bar{S}}^{*}$ is a maximum matching of $\mathcal{B}(\bar{A}, \bar{S})$, then $M_{\bar{A}, \bar{S}}^{*}=M_{\bar{S}} \cup M_{\bar{A}}$, where $M_{\bar{A}}=M_{\bar{A}, \bar{S}} \cap \mathcal{E}_{\mathcal{X}, \mathcal{X}}$ and $M_{\bar{S}}=M_{\bar{A}, \bar{S}} \cap \mathcal{E}_{\mathcal{S}, \mathcal{X}}$ are (disjoint) matchings of $\mathcal{B}(\bar{A})$ and $\mathcal{B}(\bar{S})$, respectively, and $M_{\bar{S}}$ contains the largest collection of edges incoming into a set of right-unmatched vertices of some maximum matching of $\mathcal{B}(\bar{A})$. In particular, $\mathcal{R}\left(M_{\bar{S}}\right) \subset \mathcal{U}_{R}\left(M_{\bar{A}}\right)$, where $\mathcal{U}_{R}\left(M_{\bar{A}}\right)$ is the set of right-unmatched vertices associated with the (possibly not maximum) matching $M_{\bar{A}}$.

Proof. From Lemma 1-(2), we obtain that $M_{\bar{A}}$ and $M_{\bar{S}}$ are (disjoint) matchings of $\mathcal{B}(\bar{A})$ and $\mathcal{B}(\bar{S})$, respectively. Now, recall that any set of right-unmatched vertices $\mathcal{U}_{R}$ associated with a matching of a bipartite graph comprises a set of right-unmatched vertices $\mathcal{U}_{R}^{*}$ associated with a maximum matching of that bipartite graph (Pequito et al., 2015a). Next, given that $M_{\bar{A}, \bar{S}}^{*}$ is a maximum matching of $\mathcal{B}(\bar{A}, \bar{S})$, it follows that $\mathcal{U}_{R}\left(M_{\bar{A}, \bar{S}}^{*}\right)$ comprises the lowest possible number of right-unmatched vertices. Now, to establish that $M_{\bar{S}}$ contains the largest collection of edges incoming into a set of right-unmatched vertices of a maximum matching of $\mathcal{B}(\bar{A})$, suppose by contradiction, that this is not the case. Then, there exists at least one more right-unmatched vertex in the set of right-unmatched vertices associated with a matching $M^{\prime}$ of $\mathcal{B}(\bar{A}, \bar{S})$ than in the set of right-unmatched vertices associated with a maximum matching $M_{\bar{A}, \bar{S}}^{*}$; hence, $M^{\prime}$ cannot be a maximum matching, a contradiction.

To obtain particular maximum matchings we can consider different cost structures. Therefore, we now extend the results of Lemma 1 and Lemma 2 to weighted bipartite graphs.

Lemma 3. Let $\bar{A} \in\{0,1\}^{n \times n}$ and $\bar{S} \in\{0,1\}^{n \times p}$ with $p \leq n$. Consider the weighted state-slack bipartite graph $(\bar{B}(\bar{A}, \bar{S}) ; w)$, where $\mathcal{B}(\bar{A}, \bar{S})=\mathcal{B}(\mathcal{X} \cup \mathcal{S}, \mathcal{X}, \mathcal{E} \equiv$ $\left.\left(\mathcal{E}_{\mathcal{X}, \mathcal{X}} \cup \mathcal{E}_{\mathcal{S}, \mathcal{X}}\right)\right)$, and $w: \mathcal{E} \rightarrow \mathbb{R}_{0}^{+} \cup\{\infty\}$ such that $w\left(e_{\bar{S}}\right)>w\left(e_{\bar{A}}\right)=c_{\bar{A}} \in \mathbb{R}^{+}$, with $e_{\bar{S}} \in \mathcal{E}_{\mathcal{S}, \mathcal{X}}$ and $e_{\bar{A}} \in \mathcal{E}_{\mathcal{X}, \mathcal{X}} \cdot A$ minimum weighted maximum matching $M_{\bar{A}, \bar{S}}^{*}$ of $(\mathcal{B}(\bar{A}, \bar{S}) ; w)$ is given by

$$
M_{\bar{A}, \bar{S}}^{*}=M_{\bar{A}}^{*} \cup \mathcal{E}_{\bar{S}}^{*},
$$

where $\mathcal{E}_{\bar{S}}^{*}$ consists in the largest collection of edges incoming into a set of right-unmatched vertices associated with a maximum matching $M_{\bar{A}}^{*}$ of $\mathcal{B}(\bar{A})$ and such that $\mathcal{E}_{\bar{S}}^{*}$ incurs in the lowest weight-sum among all possible collection of edges incoming into a set of right-unmatched vertices associated with a maximum matching of $\mathcal{B}(\bar{A}) . \diamond$

Proof. From Lemma 2, we have that any maximum matching of $\mathcal{B}(\bar{A}, \bar{S})$ comprises a set $\mathcal{E}_{\bar{S}}^{*} \subset \mathcal{E}_{\mathcal{S}, \mathcal{X}}$ that 
consists in the largest collection of edges incoming into a set of right-unmatched vertices associated with a maximum matching $M_{\bar{A}}^{*}$ of $\mathcal{B}(\bar{A})$. In addition, since the weights of the edges in $\mathcal{E}_{\mathcal{X}, \mathcal{X}}$ are uniform and less than the weights of the edges in $\mathcal{E}_{\mathcal{S}, \mathcal{X}}$, it follows that the edges from $\mathcal{E}_{\mathcal{X}, \mathcal{X}}$ are preferred over the edges in $\mathcal{E}_{\mathcal{S}, \mathcal{X}}$ as far as the maximum matching $M_{\bar{A}, \bar{S}}^{*}$ is concerned; consequently, the edges from $\mathcal{E}_{\mathcal{X}, \mathcal{X}}$ in $M_{\bar{A}, \bar{S}}^{*}$ are those that belong to a maximum matching $M_{\bar{A}}^{*}$ of $\mathcal{B}(\bar{A})$. By noticing that the weight-sum of all matchings of $\mathcal{B}(\bar{A})$ incur in the same cost, and a set $\mathcal{E}_{S}^{*}$ with the characteristics previously described must belong to the maximum matching $M_{\bar{A}, \bar{S}}^{*}$ of $(\mathcal{B}(\bar{A}, \bar{S}) ; w)$, the minimum cost of $M_{\bar{A}, \bar{S}}^{*}$ is achieved by considering the set $\mathcal{E}_{\bar{S}}^{*}$ incurring in the lowest weight-sum, among all possible collection of edges incoming into a set of right-unmatched vertices associated with a maximum matching of $\mathcal{B}(\bar{A})$.

By reversing the inequality between the weights of the edges between state variables and those outgoing from the slack variables, we obtain the following result.

Lemma 4. Let $\bar{A} \in\{0,1\}^{n \times n}$ and $\bar{S} \in\{0,1\}^{n \times p}$ with $p \leq n$. Consider the weighted state-slack bipartite graph $(\overline{\mathcal{B}}(\bar{A}, \bar{S}) ; w)$, where $\mathcal{B}(\bar{A}, \bar{S})=\mathcal{B}(\mathcal{X} \cup \mathcal{S}, \mathcal{X}, \mathcal{E} \equiv$ $\left.\left(\mathcal{E}_{\mathcal{X}, \mathcal{X}} \cup \mathcal{E}_{\mathcal{S}, \mathcal{X}}\right)\right)$, and $w: \mathcal{E} \rightarrow \mathbb{R}_{0}^{+} \cup\{\infty\}$ such that $w\left(e_{\bar{S}}\right)<w\left(e_{\bar{A}}\right)=c_{\bar{A}} \in \mathbb{R}^{+}$, with $e_{\bar{S}} \in \mathcal{E}_{\mathcal{S}, \mathcal{X}}$ and $e_{\bar{A}} \in \mathcal{E}_{\mathcal{X}, \mathcal{X}} . A$ minimum weighted maximum matching $M_{\bar{A}, \bar{S}}^{*}$ of $(\mathcal{B}(\bar{A}, \bar{S}) ; w)$ is given by

$$
M_{\bar{A}, \bar{S}}^{*}=M_{\bar{S}}^{*} \cup M_{\bar{A}},
$$

where $M_{\bar{S}}^{*}$ and $M_{\bar{A}}$ are as given in Lemma 2 , and $M_{\bar{S}}^{*}$ is a maximum matching of $\mathcal{B}(\bar{S})=\mathcal{B}\left(\mathcal{S}, \mathcal{X}, \mathcal{E}_{\mathcal{S}, \mathcal{X}}\right)$ whose edges incur in the lowest weight-sum among all possible maximum matchings of $\mathcal{B}(\bar{S})$.

Proof. The proof follows a similar reasoning to that in the proof of Lemma 2. In particular, notice that $M_{\bar{S}}^{*}$ is a maximum matching of $\mathcal{B}(\bar{S})$ because a weight-sum of the edges of a maximum matching of $\mathcal{B}(\bar{S})$ is smaller than that of a collection of edges of $\mathcal{B}(\bar{A}, \bar{S})$ with the same size containing edges from $\mathcal{E}_{\mathcal{X}, \mathcal{X}}$, and secondly it consists in the largest collection of edges incoming into a set of right-unmatched vertices associated with a maximum matching $M_{\bar{A}}^{*}$ of $\mathcal{B}(\bar{A})$.

Now, we present the (general) reduction of $\mathcal{P}_{1}$ and $\mathcal{P}_{2}$ to a MWMM problem in Algorithm 1. The solutions obtained are dedicated, and to obtain a solution to either problem depends on: (i) a specific weight function, and (ii) an interpretation of the minimum weighted maximum matching obtained. Later, we explain how the dedicated solutions can be used to characterize all possible solutions to $\mathcal{P}_{1}$ and $\mathcal{P}_{2}$ by recalling Remark 1 , see Remark 2 for details. Before we present the weight functions and interpretation of the minimum weighted maximum matching that lead to dedicated solutions to $\mathcal{P}_{1}$ and $\mathcal{P}_{2}$, the general reduction of $\mathcal{P}_{1}$ and $\mathcal{P}_{2}$ to a MWMM problem in Algorithm 1 can be intuitively described as follows.

\section{Algorithm 1 Solution to $\mathcal{P}_{1} / \mathcal{P}_{2}$}

Input: The structural $n \times n$ system matrix $\bar{A}$, and the vector $c$ of size $n$ comprising the cost of actuating each state variable.

Output: A solution $\bar{B}$ to $\mathcal{P}_{1} / \mathcal{P}_{2}$ comprising dedicated inputs.

1. Determine the minimum number $p$ of dedicated inputs required to ensure structural controllability (Pequito et al., 2015a).

2. Let $\mathcal{N}_{j}^{T}$, with $j=1, \cdots, \beta$, denote the non-top linked SCCs of $\mathcal{D}(\bar{A})$. Let $c_{\max }$ be the maximum real value (i.e., not considering $\infty$ ) in $c$, and consider the $n \times p$ matrix $\bar{S}$, where $p$ denotes the number of slack variables. In addition, consider $(\mathcal{B}(\bar{A}, \bar{S}) ; w)$ where $w$ is specific weight function associated with $\mathcal{P}_{1}$ and $\mathcal{P}_{2}$, as described in Theorem 2 and Theorem 3 , respectively.

3. Determine the MWMM $M^{*}$ associated with the bipartite graph $(\mathcal{B}(\bar{A}, \bar{S}) ; w)$.

4. Consider the set of indices of the state variables $\mathcal{J} \subset$ $\{1, \ldots, n\}$ obtained from interpreting the matching edges in $M^{*}$ for problem $\mathcal{P}_{1}$ and $\mathcal{P}_{2}$, as described in Theorem 2 and Theorem 3 , respectively. Set $\bar{B}=\mathbb{D}(\mathcal{J})$, where $\mathbb{D}(\mathcal{J})$ is the $n \times n$ diagonal matrix with $\mathbb{D}_{j j}=1$ if $j \in \mathcal{J}$, and $\mathbb{D}_{j j}=0$ otherwise.

5. If the weight-sum of $M^{*}$ is finite, then $(\bar{A}, \bar{B})$ is structurally controllable, and a solution to $\mathcal{P}_{1} / \mathcal{P}_{2}$ is obtained; otherwise, the problem is infeasible, i.e., there is no feasible $\bar{B}$ (with finite cost) such that $(\bar{A}, \bar{B})$ is structurally controllable.

Informal Description of Algorithm 1: The slack variables introduced, in the same number as the minimum number of state variables required to obtain a feasible dedicated input configuration, indicate through the matching edges with the state variables which state variables should be considered to achieve a feasible dedicated input configuration. Towards this goal, outgoing edges from the slack variables into the state variables are chosen such that a MWMM containing these edges exists. Next, weights are chosen such that the feasible dedicated input configuration determined incurs in minimum cost, where the state variables considered are determined from the matching edges in the MWMM. $\diamond$

To obtain the solution to $\mathcal{P}_{1}$ using Algorithm 1, consider the following result.

Theorem 2. Consider Algorithm 1, where $\bar{S}_{i, k}=1$ if $x_{i} \in \mathcal{N}^{k}$ with $k=1, \ldots, \beta$, and $\bar{S}_{i, k}=0$ otherwise; further, for $k=\beta+1, \ldots, p$ we have $\bar{S}_{i, k}=1$ for $i=$ $1, \ldots, n$; in other words, each slack variable $k=1, \ldots, \beta$ has outgoing edges to all the state variables in the $k$-th non-top linked $S C C \mathcal{N}_{k}^{T}$, whereas, for the remaining $p-\beta$ slack variables we introduce outgoing edges to all state variables. In addition, let the weight function in Step 2 to be

$$
w(e)=\left\{\begin{array}{c}
c_{\max }+1, \quad e \in \mathcal{E}_{\mathcal{X}, \mathcal{X}}, \\
c_{i}, \quad e \equiv\left(s_{j}, x_{i}\right) \in \mathcal{E}_{\mathcal{S}, \mathcal{X}}, j=1, \ldots, p,
\end{array}\right.
$$

and the interpretation of the $M W M M$ given by $M^{*}$ in Step 4 to be as follows: $\mathcal{J}=\left\{i \in\{1, \ldots, n\}:\left(s_{k}, x_{i}\right) \in\right.$ 
$\left.M^{*}, k=1, \cdots, p\right\}$. Moreover, the overall computational complexity of Algorithm 1 is $\mathcal{O}\left(n^{\omega}\right)$, where $\omega<2.373$ is the lowest exponent known associated with the complexity of multiplying two $n \times n$ matrices.

Proof. First, we notice that a solution obtained using Algorithm 1 with the proposed weight function is feasible, if the weight-sum of $M_{\bar{A}, \bar{B}}^{*}$ is finite. Because a feasible dedicated input configuration with $p$ state variables exists, where $\beta$ state variables belong in different nontop linked SCCs, and the remaining $p-\beta$ state variables correspond to right-unmatched vertices in the set of right-unmatched vertices associated with a maximum matching of the state bipartite graph $\mathcal{B}(\bar{A})$ and do not belong to the non-top linked SCCs.

Therefore, from Lemma 2 we can argue that a maximum matching of $\mathcal{B}(\bar{A}, \bar{S})$ contains edges outgoing from slack variables and ending in all right-unmatched vertices with respect to a maximum matching of $\mathcal{B}(\bar{A})$. Furthermore, there exists a maximum matching $M_{\bar{A}, \bar{S}}^{*}$ of $\mathcal{B}(\bar{A}, \bar{S})$, where all slack variables belong to matching edges in $M_{\bar{A}, \bar{S}}^{*}$. In the former case, due to the proposed construction, there is at least one edge from a slack variable to each non-top linked SCC; hence, by Theorem 1, the collection of the state variables, where the edges with origin in slack variables belonging to $M_{\bar{A}, \bar{S}}^{*}$ end, is a feasible dedicated input configuration; such a collection is also minimal since it has exactly $p$ state variables - the size of a minimal feasible dedicated input configuration.

Consequently, we aim to determine such a matching, which will be accomplished by considering a MWMM problem. More precisely, we associate a weight function $w$ as proposed. Consequently, taking $(\mathcal{B}(\bar{A}, \bar{S}) ; w)$ to be the weighted version of $\mathcal{B}(\bar{A}, \bar{S})$ with the weight function as previously described, by invoking Lemma 4 , there exists a maximum matching $M_{\bar{A}, \bar{S}}^{*}$ of $\mathcal{B}(\bar{A}, \bar{S})$, where each edge with origin in slack variables belonging to $M_{\bar{A}, \bar{S}}^{*}$ indicates which state variables should be actuated, and such that the sum of the weights of the edges in $M_{\bar{A}, \bar{S}}^{*}$ is finite. In other words, an infinite cost would correspond to the case where no feasible dedicated input configuration exists, i.e., no finite cost input matrix $\bar{B}$ can make the system structurally controllable. In summary, we obtain a minimal feasible dedicated input configuration with the lowest cost, which corresponds to a (dedicated) solution to $\mathcal{P}_{1}$.

Now, to conclude that $\bar{B}$ obtained by Algorithm 1 incurs in the minimum cost, suppose by contradiction that this is not the case. This implies that, there exists another feasible $\bar{B}^{\prime}$ leading to a smaller cost. If $\bar{B}^{\prime}$ has multiple nonzeros in the same column, given Remark 1 , there exists $\bar{B}^{\prime \prime}$ with the same cost as $\bar{B}^{\prime}$ and with at most one nonzero entry in each column such that $\left(\bar{A}, \bar{B}^{\prime \prime}\right)$ is structurally controllable. Consequently, by letting $\mathcal{D}\left(\bar{A}, \bar{B}^{\prime \prime}\right)=\left(\mathcal{X} \cup \mathcal{U}, \mathcal{E}_{\mathcal{X}, \mathcal{X}} \cup \mathcal{E}_{\mathcal{U}, \mathcal{X}}\right)$ and $\mathcal{D}(\bar{A}, \bar{S})$ to be isomorphic, and considering the weight function $w$ as in Algorithm 1, it follows by Lemma 4 that there exists a maximum matching $M^{\prime \prime}$ of $\left(\mathcal{B}(\bar{A}, \bar{S})=\left(\mathcal{X} \cup \mathcal{S}, \mathcal{E}_{\mathcal{X}, \mathcal{X}} \cup \mathcal{E}_{\mathcal{S}, \mathcal{X}}\right) ; w\right)$ containing $\mathcal{E}_{\mathcal{S}, \mathcal{X}}$. Nevertheless, this is a contradiction since it implies there exists a maximum matching $M^{\prime \prime}$ incurring in a lower cost than $M^{*}$ obtained, and used to construct $\bar{B}$.

Finally, the computational complexity follows from noticing that Step 1 can be determined by solving a MWMM (Pequito et al., 2015a). Step 2 can be computed using linear complexity algorithms. Step 3 consists in solving a MWMM. In addition, Step 4 consists of a for-loop operation which has linear complexity, as well as Step 5. Therefore, the complexity of solving the MWMM dominates, whose solution can be determined in $\mathcal{O}\left(n^{\omega}\right)$ (Mucha and Sankowski, 2004), and the result follows.

Next, we present the solution to $\mathcal{P}_{2}$ using Algorithm 1.

Theorem 3. Consider Algorithm 1, where $\bar{S}=\mathbf{1}_{n \times p}$ is the matrix with all entries equal to 1 ; in other words, each slack variable has outgoing edges to all the state variables. In addition, let the weight function in Step 2 to be

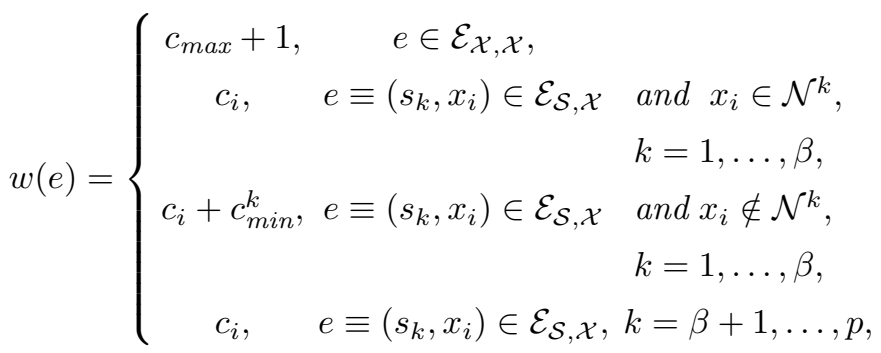

where $c_{\min }^{k}$ corresponds to the minimum cost associated with the state variables in $\mathcal{N}_{k}^{T}$. Further, let the interpretation of the MWMM given by $M^{*}$ in Step 4 to be as follows: let $M^{*}=\left\{\left(s_{k}, x_{\sigma(k)}\right): k=1, \cdots, p\right\}$ where $\sigma($. is a permutation of the state variables indices. Consider $\Theta=\bigcup_{k=1, \ldots, p} \Omega_{k}$, where

$$
\Omega_{k}=\left\{\begin{array}{l}
\left\{x_{\sigma(k)}\right\}, \text { if } x_{\sigma(k)} \in \mathcal{N}_{k}^{T}, \\
\left\{x_{\sigma(k)}, x_{\text {min }}^{k}\right\}, \text { if } x_{\sigma(k)} \notin \mathcal{N}_{k}^{T},
\end{array}\right.
$$

with $x_{\text {min }}^{k}$ a state variable in $\mathcal{N}_{k}^{T}$ with the minimum cost, and take $\mathcal{J}=\left\{i \in\{1, \ldots, n\}: x_{i} \in \Theta\right\}$. Moreover, the overall computational complexity is of $\mathcal{O}\left(n^{\omega}\right)$, where $\omega<2.373$ is the lowest exponent known associated with the complexity of multiplying two $n \times n$ matrices.

Proof. The proof follows similar steps to those in Theorem 2, where for the feasibility and minimality we need to notice that because of the (potential) double-role of the state variables in a minimal feasible input configuration (i.e., state variables in a non-top linked SCC and right-unmatched vertices), we may be able to further reduce the cost by considering two state variables instead of one playing a double role used in the construction of a minimal feasible dedicated input configuration (associated with a solution to $\mathcal{P}_{1}$ ), while retaining the feasibility. 
Finally, in Remark 2 we characterize all possible solutions to $\mathcal{P}_{1}$ and $\mathcal{P}_{2}$ given a dedicated solution obtained with Algorithm 1.

Remark 2. Now, consider $\left(\mathcal{B}\left(\bar{A}, \bar{B}^{e}\right)=\left(\mathcal{X} \cup \mathcal{S}, \mathcal{E}_{\mathcal{X}, \mathcal{X}} \cup\right.\right.$ $\left.\left.\mathcal{E}_{\mathcal{U}, \mathcal{X}}\right), w^{\prime}\right)$ where $\bar{B}^{e}$ contains only the non-zero columns of $B$ obtained from Algorithm 1, i.e., the effective inputs, and $w^{\prime}$ is given as follows:

$$
w^{\prime}(e)=\left\{\begin{array}{l}
1, e \in \mathcal{E}_{\mathcal{X}, \mathcal{X}}, \\
2, e \in \mathcal{E}_{\mathcal{U}, \mathcal{X}} .
\end{array}\right.
$$

Therefore, considering $\left(\mathcal{B}\left(\bar{A}, \bar{B}^{e}\right) ; w\right)$ and using Lemma 3 , a $M W M M$ comprises the edges from $\mathcal{E}_{\mathcal{U}, \mathcal{X}}$ with endpoints in the state variables that belong to the set of right-unmatched vertices $\mathcal{U}_{R}\left(M_{A}^{*}\right)$ associated with a maximum matching $M_{\bar{A}}^{*}$ of $\mathcal{B}(\bar{A})$. Consequently, from Remark 1 and the dedicated solution obtained with Algorithm 1, we can further obtain a non-dedicated solution to $\mathcal{P}_{1} / \mathcal{P}_{2} ;$ more precisely, one requires $m$ distinct inputs, where $m$ is the number of right-unmatched vertices $\mathcal{U}_{R}\left(M_{\bar{A}}^{*}\right)$, assigned to those state variables in $\mathcal{U}_{R}\left(M_{\bar{A}}^{*}\right)$ and some input (potentially the same) must be assigned to the remaining state variables required to ensure structural controllability (identified by the dedicated solution). Finally, because the cost is associated with each directly actuated state variable and is independent of the labeled input variable, the (overall) costs attained by the dedicated and non-dedicated solutions are the same. $\diamond$

\section{Illustrative Example}

Consider the state digraph depicted in Figure 1 and the manipulating costs $c=\left[\begin{array}{lllllll}50 & \infty & 10 & 10 & 1 & 10 & 20\end{array}\right]$. The solutions to $\mathcal{P}_{1}$ and $\mathcal{P}_{2}$ are now presented, resorting to Algorithm 1 with the additional constraints as in Theorem 2 and Theorem 3, respectively. In Step 1 of Algorithm 1, we obtain that the minimum number of dedicated inputs required to ensure structural controllability is $p=2$. Thus, two slack variables, denoted by $s_{1}, s_{2}$, are introduced. From each slack variable, new edges to the state variables are introduced to obtain the bipartite graph $(\mathcal{B}(\bar{A}, \bar{S}) ; w)$ as described in Algorithm 1 - Step 2; see Figure 1 a) and Figure 1 b) for the associated weighted digraphs considering the constraints in Theorem 2 and Theorem 3, respectively. The MWMMs obtained in Step 3 to address $\mathcal{P}_{1}$ and $\mathcal{P}_{2}$ are $M^{1}=$ $\left\{\left(s_{1}, x_{1}\right),\left(s_{2}, x_{6}\right),\left(x_{2}, x_{2}\right),\left(x_{1}, x_{3}\right),\left(x_{3}, x_{4}\right),\left(x_{4}, x_{5}\right)\right.$, $\left.\left(x_{5}, x_{7}\right)\right\}$ and $M^{2}=\left\{\left(s_{1}, x_{4}\right),\left(s_{2}, x_{6}\right),\left(x_{2}, x_{1}\right),\left(x_{1}, x_{3}\right)\right.$, $\left.\left(x_{3}, x_{2}\right),\left(x_{6}, x_{5}\right),\left(x_{5}, x_{7}\right)\right\}$, respectively. Subsequently, from Step 4 we obtain $\mathcal{J}^{1}=\{1,6\}$ and $\mathcal{J}^{2}=\{3,4,6\}$, where the associated actuation cost is 60 and 30, respectively. Notice that the sum of weights in $M^{1}$ and $M^{2}$ is finite, hence, $\mathbb{D}\left(\mathcal{J}^{1}\right)$ and $\mathbb{D}\left(\mathcal{J}_{2}\right)$ are (dedicated) solutions to $\mathcal{P}_{1}$ and $\mathcal{P}_{2}$, respectively. In addition, notice that by actuating more state variables it is possible to further minimize the overall cost; in particular, this is due to the fact that $x_{1}$ is a state variable with double role, i.e., besides being a right-unmatched vertex, it is also a variable in a non-top linked SCC.
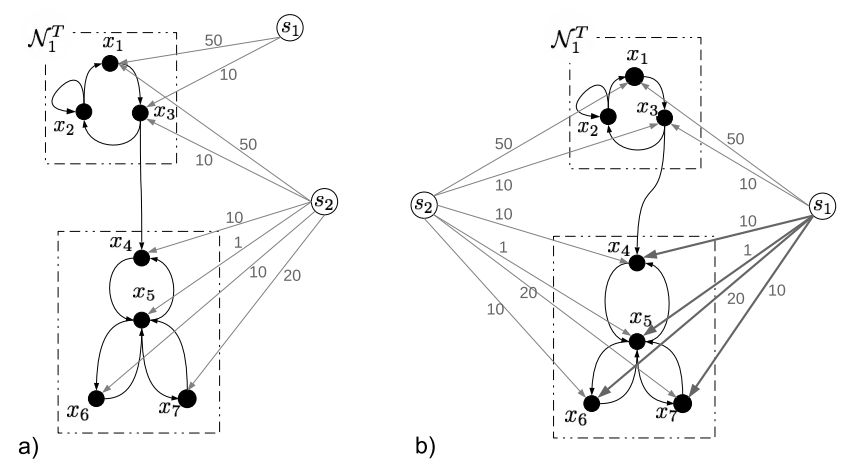

Fig. 1. An illustrative example of a digraph $\mathcal{D}(\bar{A})$, where the SCCs are inscribed in the dashed boxes and the non-top linked SCC labeled by $\mathcal{N}_{1}^{T}$. The edges' costs are depicted by gray labels, the edges that are not depicted have $\infty$ cost, and the edges between state variables have cost equal to 51 . In this figure, we present the reduction Step 2 of Algorithm 1; more precisely, in a) we used the weight function in Theorem 2 , and in $\mathrm{b}$ ) the one associated with the weight function in Theorem 3.

\section{Conclusions and Further Research}

In this paper, we provided a systematic method with polynomial complexity (in the number of the state variables) in order to obtain minimal cost placements of actuators ensuring structural controllability of a given LTI system. The proposed solutions hold under arbitrary non-homogeneous positive assignment costs for the manipulation of the state variables. By duality, the results extend to the corresponding structural observability output design under cost constraints. The non-homogeneity of the allocation cost makes the framework particularly applicable to input (output) topology design in largescale dynamic infrastructures, such as power systems, which consist of a large number of heterogeneous dynamic components with varying overheads for controller (sensor) placement and operation. Future research may focus on the development of solutions to actuator/sensor placement under more general assumptions on the cost.

\section{References}

Assadi, S., Khanna, S., Li, Y. and Preciado, V. M. (2015), 'Complexity of the minimum input selection problem for structural controllability', IFAC-PapersOnLine 48(22), 70 - 75. 5th IFAC Workshop on Distributed Estimation and Control in Networked Systems NecSys, 10-11 September 2015.

Begg, D. W. and Liu, X. (2000), 'On simultaneous optimization of smart structures - part II: Algorithms and examples', Computer Methods in Applied Mechanics and Engineering 184(1), 25 - 37.

Chmielewski, D. J., Peng, J.-K. and Manthanwar, A. M. (2002), Convex methods in actuator placement, in 'Proceedings of the American Control Conference', Vol. 6, pp. $4309-4314$ vol.6.

Clark, A., Alomair, B., Bushnell, L. and Poovendran, R. (2014), 'Minimizing convergence error in multi-agent systems via leader selection: A supermodular optimization approach', IEEE Transactions on Automatic Control 59(6), 1480-1494. 
Clark, A. and Poovendran, R. (2011), A submodular optimization framework for leader selection in linear multiagent systems, in '50th IEEE Conference on Decision and Control and European Control Conference', pp. 36143621 .

Commault, C. and Dion, J.-M. (2013), 'Input addition and leader selection for the controllability of graph-based systems.', Automatica 49(11), 3322-3328.

Cormen, T. H., Stein, C., Rivest, R. L. and Leiserson, C. E. (2001), Introduction to Algorithms, 2nd edn, McGraw-Hill Higher Education.

Dion, J.-M., Commault, C. and der Woude, J. V. (2003), 'Generic properties and control of linear structured systems: a survey.', Automatica pp. 1125-1144.

Duan, R. and Pettie, S. (2014), 'Linear-time approximation for maximum weight matching', J. ACM 61(1), 1:1-1:23.

Fahroo, F. and Demetriou, M. A. (2000), 'Optimal actuator/sensor location for active noise regulator and tracking control problems', Journal of Computational and Applied Mathematics 114(1), 137 - 158.

Frecker, M. I. (2003), 'Recent advances in optimization of smart structures and actuators', Journal of Intelligent Material Systems and Structures 14(4-5), 207-216.

Hespanha, J. P. (2009), Linear Systems Theory, Princeton Press, Princeton, New Jersey.

Kuhn, H. W. (1955), 'The Hungarian Method for the Assignment Problem', Naval Research Logistics Quarterly 2(12), 83-97.

Lin, C. (1974), 'Structural controllability', IEEE Transactions on Automatic Control (3), 201-208.

Lin, F., Fardad, M. and Jovanović, M. R. (2014), 'Algorithms for leader selection in stochastically forced consensus networks', IEEE Trans. Automat. Control 59(7), 1789-1802.

Liu, Y.-Y., Slotine, J.-J. and Barabási, A.-L. (2011), 'Controllability of complex networks', Nature 473(7346), 167173.

Mucha, M. and Sankowski, P. (2004), Maximum matchings via gaussian elimination, in 'Proceedings. 45th Annual IEEE Symposium on Foundations of Computer Science, 2004', pp. 248-255.

Munkres, J. (1957), 'Algorithms for the Assignment and Transportation Problems', Journal of the Society for Industrial and Applied Mathematics 5(1), 32-38.

Murota, K. (2009), Matrices and Matroids for Systems Analysis, 1st edn, Springer Publishing Company, Incorporated.

Olshevsky, A. (2014), 'Minimal controllability problems', IEEE Transactions on Control of Network Systems 1(3), 249-258.

Olshevsky, A. (2015), Minimum input selection for structural controllability, in 'Proceedings of the American Control Conference (ACC)', pp. 2218-2223.

Padula, S. L. and Kincaid, R. K. (1999), Optimization strategies for sensor and actuator placement, Technical report, National Aeronautics and Space Administration Langley Research Center, Langley, Virginia 23681.

Pasqualetti, F., Zampieri, S. and Bullo, F. (2014), 'Controllability metrics, limitations and algorithms for complex networks', IEEE Transactions on Control of Network Systems 1(1), 40-52.

Pequito, S., Kar, S. and Aguiar, A. (2013a), Optimal cost actuator/sensor placement for large scale linear timeinvariant systems: A structured systems approach, in 'Proceedings of European Control Conference', pp. 815-820.

Pequito, S., Kar, S. and Aguiar, A. (2013b), A structured systems approach for optimal actuator-sensor placement in linear time-invariant systems, in 'Proceedings of the American Control Conference', pp. 6108-6113.

Pequito, S., Kar, S. and Aguiar, A. P. (2013c), Minimum cost input-output and control configuration selection: A structural systems approach, in 'Proceedings of IEEE 52nd Annual Conference on Decision and Control', pp. 4895-4900.

Pequito, S., Kar, S. and Aguiar, A. P. (2015a), 'A framework for structural input/output and control configuration selection of large-scale systems', IEEE Transactions on Automatic Control. To Appear .

Pequito, S., Kar, S. and Aguiar, A. P. (2015b), 'On the complexity of the constrained input selection problem for structural linear systems', Automatica 62, 193 - 199.

Ramos, G., Pequito, S., Kar, S., Aguiar, A. P. and Ramos, J. (2014), 'On the Exact Solution of the Minimal Controllability Problem', ArXiv e-prints .

Reinschke, K. J. (1988), Multivariable control: a graph theoretic approach, Vol. 108 of Lecture Notes in Control and Information Sciences, Springer-Verlag.

Ruths, J. and Ruths, D. (2014), 'Control Profiles of Complex Networks', Science 343(6177), 1373-1376.

Siljak, D. D. (2007), Large-Scale Dynamic Systems: Stability and Structure, Dover Publications.

Summers, T., Cortesi, F. and Lygeros, J. (2015), 'On submodularity and controllability in complex dynamical networks', IEEE Transactions on Control of Network Systems $\mathbf{P P}(99), 1-1$

Tzoumas, V., Rahimian, M., Pappas, G. and Jadbabaie, A. (2015), 'Minimal actuator placement with bounds on control effort', IEEE Transactions on Control of Network Systems $\mathbf{P P}(99), 1-1$. 